\title{
Impaired Spatial Performance in Rats with Retrosplenial Lesions: Importance of the Spatial Problem and the Rat Strain in Identifying Lesion Effects in a Swimming Pool
}

\author{
K. Troy Harker and lan Q. Whishaw \\ Canadian Center for Behavioral Neuroscience, Lethbridge, Alberta, T1K 3M4, Canada
}

Behavioral, electrophysiological, and anatomical evidence suggests that retrosplenial (RS) cortex (areas RSA and RSG) plays a role in spatial navigation. This conclusion has been questioned in recent work, suggesting that it is damage to the underlying cingulum bundle (CG) (areas CG and IG), and not RS, that disrupts spatial place learning (Aggleton et al., 2000).

We revisited this issue by comparing Long-Evans rats, the strain used in studies that report RS deficits, to Dark Agouti rats, the strain in which no $\mathrm{RS}$ deficit has been reported. Rat groups with RS, RS + CG, or no lesion were tested on a place task in a swimming pool, a test of nonspatial and spatial learning, and a matching-to-place task, a relatively selective test of spatial learning. Long-Evans rats given RS and RS + CG lesions, either before or after training on the two tasks, were impaired on both tasks, a deficit not attributable to impaired visual acuity. Control Dark Agouti rats and RS Dark Agouti rats, although not different on the place task, were both significantly impaired relative to Long-Evans rats. The RS Dark Agouti group, however, was also impaired on the matching-to-place task.

Thus, we show that RS cortex is part of an extended neural circuit involved in spatial behavior in both Long-Evans and Dark Agouti rats, but its role in the place task may be masked by an innate nonspatial deficit in Dark Agouti rats. The results are discussed in relation to the importance of assessing spatial learning with appropriate spatial tests, the problems of interpretation posed by rat strain differences, and the role of retrosplenial cortex in spatial behavior.

Key words: cingulum bundle; Dark Agouti rat; learning and memory; Long-Evans rat; matching-to-place learning; Morris water task; place learning; posterior cingulate; retrosplenial cortex; spatial navigation; strain differences
Evidence suggests that retrosplenial (RS) cortex (areas RSA and RSG) plays a role in spatial behavior. Magnetic resonance imaging studies show that there is RS activation during spatial problem solving (Mesulam et al., 2001) and that damage to RS cortex results in impaired spatial problem solving (Maguire, 2001). Behavioral studies have also shown impairments in a variety of spatial navigation tasks after RS lesions (Pohl, 1973; DeRenzi, 1982; Pandya and Yeterian, 1984; Sutherland et al., 1988; Kolb et al., 1994; Wozniak et al., 1996; Ennaceur et al., 1997; Maaswinkel et al., 1999; Cooper and Mizumori, 2001; Whishaw et al., 2001). Single-cell recording studies in freely moving animals demonstrate RS cortex cells are responsive to an animal's orientation, its spatial location, and spatial movements (Chen et al., 1994a,b; Cho and Sharp, 2001). Anatomical studies indicate that there are reciprocal connections between RS cortex and neocortex and between RS cortex and a number of structures in the hippocampal formation, including the subiculum, the entorhinal and perirhinal cortices, and area CA3 of the hippocampus proper (Vogt and Miller, 1983; Pakhomova and Akopian, 1985; Wyss and Van Groen, 1992). Taken together, these studies support a role for RS in bridging neocortical and limbic structures involved in spatial

\footnotetext{
Received Sept. 24, 2001; revised Nov. 13, 2001; accepted Nov. 19, 2001.

This work was supported by the Natural Sciences and Engineering Research Council of Canada, The Canadian Institute for Health Research, and the University of Lethbridge. Special thanks to Glen Prusky for consulting and assisting with the Visual Acuity testing, Bogdan Gorny for assistance with behavioral testing, and Dawn Danka for assistance with histology.

Correspondence should be addressed to Dr. Ian Q. Whishaw, Canadian Center for Behavioral Neuroscience, University of Lethbridge, 4401 University Drive, Lethbridge, Alberta, Canada T1K 3M4. E-mail: Whishaw@uleth.ca.

Copyright (ㄷ) 2002 Society for Neuroscience $0270-6474 / 02 / 221155-10 \$ 15.00 / 0$
}

navigation. Thus, damage to RS may result in spatial impairments by way of a disconnection (Geschwind, 1965).

The cingulum bundle (CG), lying directly beneath the RS, has also been linked anatomically to the hippocampal formation as part of the Papez (1937) circuit. Given contemporary evidence that the hippocampal formation has spatial functions, this pathway may also be involved in spatial behavior. Recent lesion studies have supported this suggestion (Neave et al., 1997; Warburton et al., 1998; Aggleton et al., 2000). Indeed, these same studies suggest that it is only the CG and not the RS that has spatial functions. The authors of these studies have proposed that the spatial place learning deficits observed in previous lesion studies resulted from inadvertent damage to the underlying CG accompanying an RS lesion and not from damage to RS itself.

There are three differences between those studies that report that cingulate cortex has spatial functions (Sutherland et al., 1988; Palmer et al., 1993; Whishaw et al., 2001) and those studies that fail to confirm this (Warburton et al., 1998; Aggleton et al., 2000). First, studies reporting no deficit used cell-specific neurotoxic lesions that were somewhat smaller than the suction ablations used in studies reporting deficits. Thus, differences in cell versus fiber damage and/or lesion size may have contributed to the difference in experimental findings. This explanation is unlikely to account for contradictory claims because crossed suction and neurotoxic lesions have been shown to impair spatial performance (Sutherland and Hoesing, 1993). Second, studies reporting no deficit used the swimming pool place task in which a rat learns to swim to a single location, whereas studies reporting a deficit used both the place task and a matching-to-place task, in which a rat learns a number of place locations. The former task is sensi- 
tive to both spatial and nonspatial deficits (Cain and Saucier, 1996), whereas the latter task is a more selectively spatial task (Whishaw, 1985b). Third, the studies failing to report RS deficits on spatial tasks used the Dark Agouti rat strain, whereas studies that report RS deficits use the Long-Evans rat strain. An examination of the acquisition curves produced by the different rat strains in the two sets of studies suggest that the Long-Evans rat strain displays superior spatial learning to Dark Agouti rats. Before the idea that the RS has spatial functions is dismissed, the possibility that task and/or strain differences is responsible for the difference in experimental results must be examined.

Our objective in the present study is to revisit the role of RS in spatial navigation by: (1) comparing the performance of rats with selective suction lesions of the RS to the performance of rats with suction lesions of both RS and CG, (2) assessing the performance of the animals on the place task and the matching-to-place task, and (3) comparing the effects of the lesions on the place task and the matching-to-place task in both Long-Evans and Dark Agouti rat strains.

\section{MATERIALS AND METHODS}

Subjects. Fifty-four male Long-Evans rats (University of Lethbridge vivarium) $90 \mathrm{~d}$ old, weighing between 260 and $490 \mathrm{gm}$, and 15 male Dark Agouti rats (Bantin and Kingman Universal, Fremont, CA) $\sim 90 \mathrm{~d}$ old, weighing between 190 and $230 \mathrm{gm}$, were used in the experiments. For all experiments, subjects were housed in groups of four or five individuals in hanging wire mesh cages. Room temperature was maintained at $20-21^{\circ} \mathrm{C}$, and lighting was on a $12 \mathrm{hr}$ light/dark cycle (8:00 A.M. 8:00 P.M.). Food and water were provided ad libitum. The subjects either received a retrosplenial cortex suction lesion, a combined retrosplenial cortex and cingulum bundle suction lesion, or no lesion.

Surgery. For all experiments the rats were anesthetized with sodium pentobarbital $(58.5 \mathrm{mg} / \mathrm{kg})$. The cortex was exposed by removing a long piece of skull $2 \mathrm{~mm}$ wide on either side of the midline, such that a strip of bone $\sim 2 \mathrm{~mm}$ wide remained over the sagittal sinus. The dura was incised with a number 11 scalpel. For the retrosplenial cortex lesions, the pia matter was wiped away along with the blood vessels, and using suction, the superficial gray matter was gently removed. The lesion did not penetrate to the underlying white matter, the cingulum bundle, or the underlying hippocampus. For the combined retrosplenial cortex and cingulum bundle lesions, the ablation included all of the gray matter, and when the underlying white matter was visualized, the dorsally protruding cingulum bundle was removed. The lesion did not include the corpus callosum or hippocampus. After hemostasis, the skin was sutured.

Histology. At the completion of the experiments, the rats were anesthetized and perfused intracardially with $0.9 \%$ buffered saline followed by $4 \%$ paraformaldehyde (PFA) and $14 \%$ saturated picric acid (PA) in $0.1 \mathrm{M} \mathrm{PO}_{4}$ buffer, $\mathrm{pH}$ 6.9. The brains were weighed and stored in the PFA-PA solution for at least $48 \mathrm{hr}$. The brains were then cut at $50 \mu \mathrm{m}$ on a Vibratome (TPI Inc, St. Louis, MO). Every tenth section was mounted and stained with cresyl violet.

Swimming pool apparatus. The swimming pool was located in a test room $(287-\mathrm{cm}$-wide $\times 533$-cm-long $\times 244$-cm-high) in which many cues, including counters, cupboards, posters, etc., were present. A $156-\mathrm{cm}-$ diameter and 46-cm-high, round white swimming pool positioned $14 \mathrm{~cm}$ above the floor, was filled to a depth of $25 \mathrm{~cm}$ with $21-22^{\circ} \mathrm{C}$ water that was made opaque by the addition of $750 \mathrm{~cm}^{3}$ of powdered milk (Sutherland et al., 1983). A clear Plexiglas platform with an $11 \mathrm{~cm}^{2}$ top could be placed in the pool so that the top of the platform was located $1 \mathrm{~cm}$ below the surface of the water, where it was not visible to a viewer on the surface of the water. The surface of the platform was serrated, so that the rats could obtain purchase as they climbed onto it. The performance of the animals in the swimming pool was tracked using a video cameracomputer-based tracking system (San Diego Instruments, San Diego, CA) that plots the rats' swimming latency, swim trajectory, swimming distance, swimming accuracy, and swimming heading. The results were analyzed using ANOVA for repeated measures (Winer, 1962).

Place task. Animals were tested two trials per day for 10 consecutive days, with the platform always located in the center of the southwest quadrant of the swimming pool (Morris et al., 1982). A trial consisted of placing a rat by hand into the water, facing the wall of the pool, at one of four starting positions (north, south, east, and west) around the perimeter of the pool. The four different start positions were distributed equally among all the subjects on each trial, with the order of start positions for any given subject occurring in a random manner. If on a particular trial a rat found the platform, it was permitted to remain on the platform for $10 \mathrm{sec}$. If after $90 \mathrm{sec}$ the rat failed to find the platform, it was then guided to the platform and permitted to remain there for $10 \mathrm{sec}$. At the end of the trial the rat was returned to a holding cage, and $\sim 10-20$ min elapsed before beginning the next trial. After two trials the animals were returned to their home cages, and the same procedure was repeated the next day. Measures of swim latency (time to find and mount the escape platform), swim distance, and swimming error were recorded. Swimming error was measured as the inability of a rat to swim in a relatively direct path from the start position to the location of the hidden platform, (Whishaw, 1985a). A correct score (assigned a value of 0) was obtained when the subject swam directly to the platform while remaining within an 18 -cm-wide corridor, extending from the start location to the platform. Any deviation from a direct swim in a relatively straight line within the corridor resulted in an incorrect score (given a value of 1).

Probe trial. On the eleventh day of testing, the rats were given a probe trial (Sutherland et al., 1983). For the probe trial the platform was removed from the tank, and the animal was allowed to swim for $60 \mathrm{sec}$. Probe trials were analyzed using a preference analysis (Brown et al., 2000). The quadrant in which the platform had been located during previous trials was designated as the target quadrant $(T)$. The swim times in the remaining three quadrants $(A, B, C)$ were then subtracted from the swim time in the target quadrant, and the resultant scores were added, and their average was derived according to the following formula: probe preference score $=((T-A)+(T-B)+(T-C)) / 3$.

Swimming error during the probe trial was measured as the inability of a rat to swim in a relatively direct path from the start position to the now vacant location of the hidden platform, which was removed for the probe trial (Whishaw, 1985a). A correct score (assigned a value of 0) was obtained when the subject swam directly to the platform while remaining within an 18-cm-wide corridor, extending from the start location to the platform. Any deviation from a direct swim in a relatively straight line within the corridor resulted in an incorrect score (given a value of 1).

Matching-to-place task. Animals were tested two trials per day for 5 consecutive days, with the platform moving to a new location each day (Whishaw, 1985b). The starting position for a given subject remained the same for both trials on a given day. Again the four start positions occurred in a random order for a given animal and were equally distributed among the subjects. The rats were placed into the pool in the same manner as for the place task. During the matching-to-place task, however, the rats were required to swim until they found the platform, where they remained for $10 \mathrm{sec}$, and were then placed in a holding cage for 20 sec before beginning trial 2 .

Visual acuity gratings test. Long-Evans control rats $(n=3)$, LongEvans retrosplenial rats $(n=6)$, and Dark Agouti control rats $(n=4)$ were tested in a water based Y-maze where the correct side (side containing the escape platform) was cued by the presence of black and white gratings (Prusky et al., 2000). The animals were tested at consecutively higher spatial frequencies of the gratings until they failed to meet a criterion of $80 \%$ correct choices.

Procedure. Three experiments were conducted

$R S$ lesions versus $R S+C G$ lesions. Naive Long-Evans rats were given either a RS suction lesion $(n=8)$ or a RS + CG suction lesion $(n=9)$ and compared with naive Long-Evans control rats $(n=10)$ on the place task, the probe trial, and the matching to place task.

Pretraining on the place and matching-to-place tasks. Two groups of animals, a Long-Evans control group $(n=11)$ and a Long-Evans RS group $(n=9)$, were used to assess the contributions of possible nonspatial impairments that may result from RS surgery. The groups were trained on the place task, given the probe trial, and trained on the matching-to-place task before RS surgery, and then the same training was given after surgery.

Long-Evans and Dark Agouti strain comparison. Three groups of animals, Dark Agouti RS rats $(n=6)$, Dark Agouti control rats $(n=9)$, and Long-Evans control rats $(n=6)$, were used to compare strain differences on the place task, the probe trial, and the matching-to-place task. Nonspatial errors were also monitored during the place task (Saucier et al., 1996). These behaviors included diving behavior (diving below the surface of the water during a trial), floating (periods of no swimming lasting three seconds or greater), platform deflections (failing to detect the platform upon contact), mounting error (a delay of one second or 

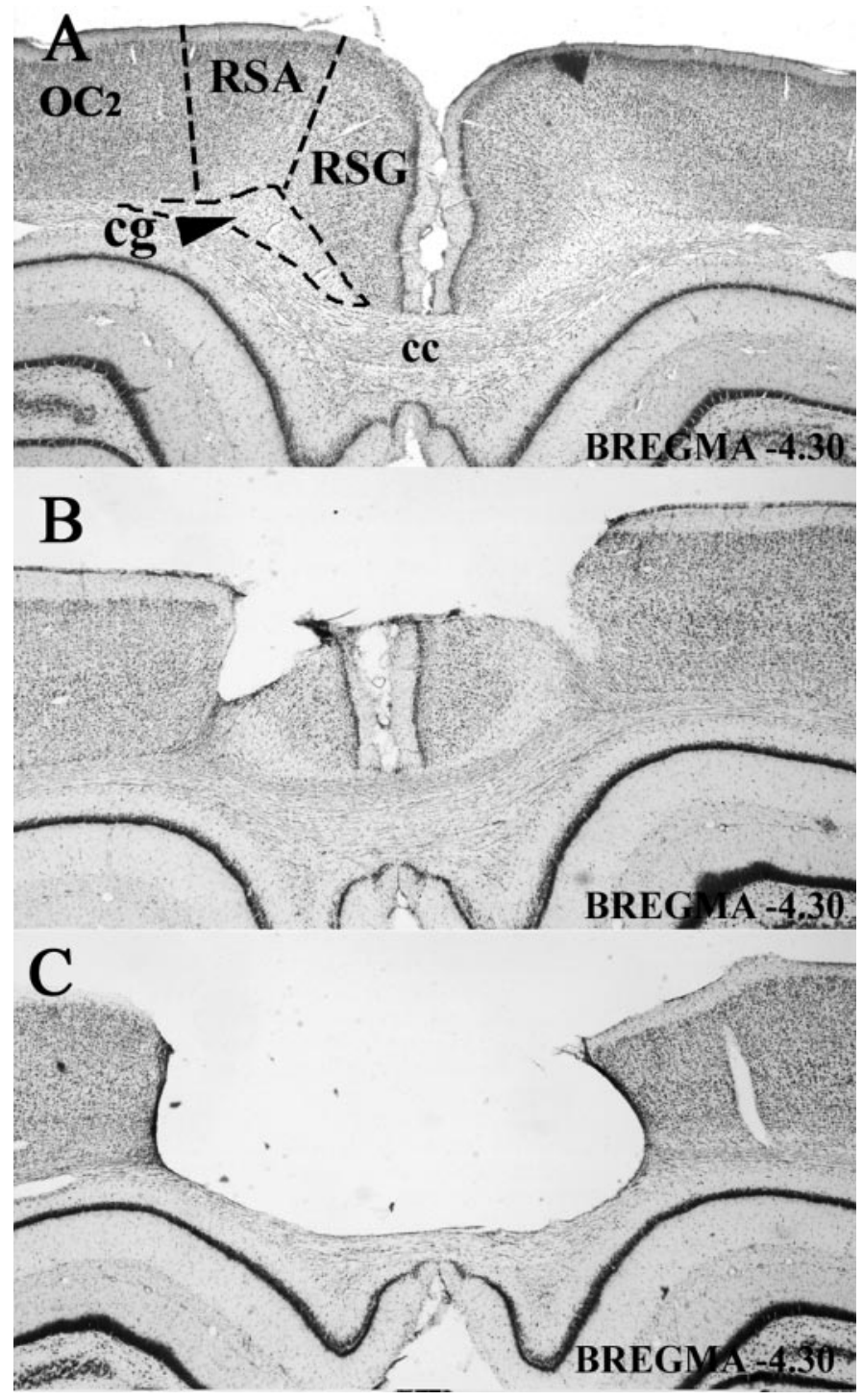

Figure 1. Photomicrographs in coronal view (approximately bregma $-4.30)$ of a representative control rat $(A)$, a representative retrosplenial rat $(B)$, and a representative retrosplenial + cingulum bundle rat $(C)$.

greater in mounting the platform upon contact), and jumping (jumping off the escape platform). Each instance of any nonspatial error was given a score of 1 ; nonspatial scores were summed across all errors for each group and analyzed using an ANOVA.

\section{RESULTS}

\section{Histological results}

The RS lesions were not as extensive as typical suction ablation lesions of this area (Sutherland et al., 1988; Whishaw et al., 2001). The lesions were, however, selective to posterior cingulate cortex with no apparent damage to the underlying cingulum bundle, corpus callosum, or hippocampus, as illustrated in Figure $1 B$.

The combined RS + CG lesions were more extensive in the removal of retrosplenial cortex yet still resulted in no apparent damage to the underlying hippocampus (Fig. 1C).

\section{Experiment: RS lesions versus RS + CG lesions}

Place task

The control rats showed a rapid decrease in latency to find the platform, such that by the fifth trial, they were performing near an asymptotic level of accuracy (Fig. 2). The RS and the RS + CG groups also demonstrated an improved performance in reaching the hidden escape platform, although both groups were impaired relative to controls. Because neither the RS nor the RS + CG groups appeared to be performing at asymptotic levels after 10 trials, all the groups were given a further 10 trials to the same location. A repeated measures (one within, one between) ANOVA for the measure of latency showed a significant group difference $\left(F_{(2,24)}=14.842 ; p<0.05\right)$, a significant trial effect $\left(F_{(19,456)}=23.044 ; p<0.05\right)$, and a significant group $\times$ trial interaction $\left(F_{(38,456)}=2.288 ; p<0.05\right)$. A Student-NewmanKeuls $(\mathrm{SNK})$ post hoc test $(p<0.05)$ gave significant group differences: control $<\mathrm{RS}$, control $<\mathrm{RS}+\mathrm{CG}$, and $\mathrm{RS}=\mathrm{RS}+$ CG. A repeated measures (one within, one between) ANOVA for swim distance reflected the analysis of latency with a significant group effect $\left(F_{(2,24)}=18.658 ; p<0.05\right)$, a significant trial effect $\left(F_{(19,456)}=18.785 ; p<0.05\right)$, and a significant group $\times$ trial interaction $\left(F_{(19,456)}=2.337 ; p<0.05\right)$. An SNK post hoc test $(p<0.05)$ showed that the two experimental groups were both significantly different from the control group, but not from each other: control $<\mathrm{RS}=\mathrm{RS}+\mathrm{CG}$. The control subjects also made fewer swimming errors en route to the platform. A repeated measures (one within, one between) ANOVA for swimming error showed a significant group difference $\left(F_{(2,24)}=13.127 ; p<0.05\right)$ with an SNK post hoc test showing the differences to be: control $<\mathrm{RS}=\mathrm{RS}+\mathrm{CG}$.

The average swimming speed (swim distance/latency) for each group of rats was also compared to rule out the possibility that the longer latencies for the experimental groups were the result of a simple motor deficit affecting swimming. A repeated measures (one within, one between) ANOVA for swimming speed showed no significant difference between any of the groups (control: mean $=35.978 \mathrm{~cm} / \mathrm{sec} ; \mathrm{SE}=0.614 ; \mathrm{RS}:$ mean $=36.732 \mathrm{~cm} /$ sec; $\mathrm{SE}=0.461 ; \mathrm{RS}+\mathrm{CG}$ : mean $=39.226 \mathrm{~cm} / \mathrm{sec} ; \mathrm{SE}=0.490$; $\left.F_{(2,24)}=1.869 ; p>0.05\right)$.

\section{Probe test}

An ANOVA for the target quadrant preference measure on the probe trial (Fig. 3A) showed no significant group differences. There was, however, a group effect for the number of target crossings $\left(F_{(2,24)}=4.167 ; p<0.05\right)$ (Fig. $\left.3 B\right)$. An SNK post hoc test revealed the group differences as: $\mathrm{RS}>\mathrm{RS}+\mathrm{CG}=$ control. That the RS group performed more target crossings is somewhat surprising, but not entirely unexpected. Brain damaged subjects have previously been shown to spend significant amounts of time in the target quadrant on a probe trial (Whishaw and Jarrard, 1995). Furthermore, it is possible that a strategy of circling in the correct quadrant will inadvertently produce elevated scores on the measure of target crossings. Thus, in the context of the other behavioral measures, it is more likely that the elevated target crossing scores are the result of a search strategy than an enhancement of spatial learning. There were no significant group differences for the number of direct swimming errors (Fig. 3C).

\section{Matching-to-place task}

Optimal performance on the matching-to-place task is characterized by the ability of a subject to learn the new location of the hidden platform in one trial. This is demonstrated behaviorally in normal subjects by elevated trial 1 latencies (an indication of searching out the previous days' location of the hidden platform) followed by significantly reduced trial 2 latencies (an indication of having learned the new platform location). A repeated measures 


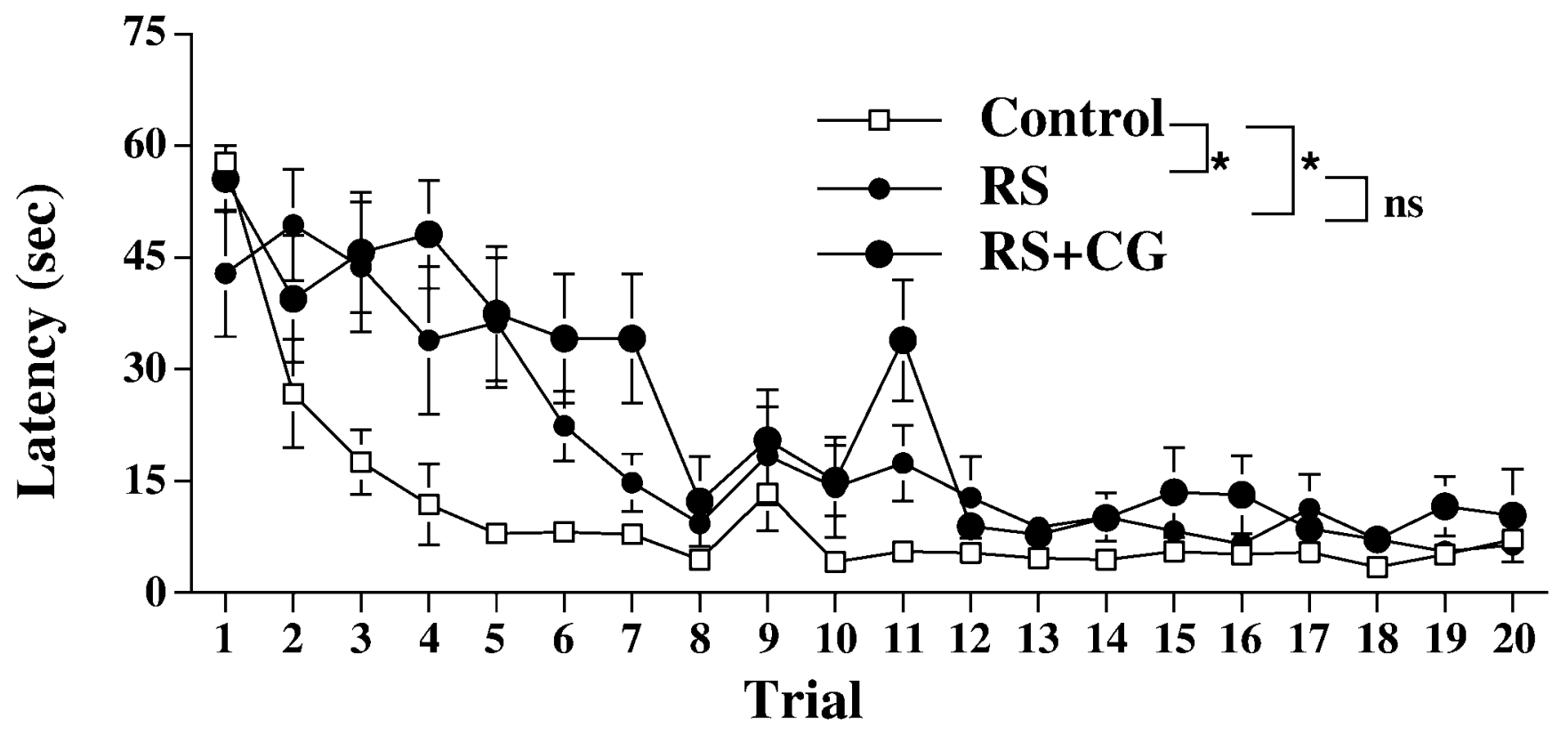

Figure 2. Latency (mean and SE) per trial by control, retrosplenial $(R S)$, and retrosplenial + cingulum bundle $(R S+C G)$ rats over 20 trials on a place task in a swimming pool. $* p<0.05$.

(two within, one between) ANOVA for the measure of latency on the matching to place task showed no significant group effect, but did show a significant effect of trial $\left(F_{(1,24)}=45.291 ; p<0.05\right)$ and a significant group $\times$ trial interaction $\left(F_{(2,24)}=5.615 ; p<\right.$ $0.05)$. These findings of no overall group effect accompanied by a trial effect and group $\times$ trial interaction prompted a further analysis of the group $\times$ trial interaction. A repeated measures (one within, one between) ANOVA for the trial 1 latencies showed no significant group differences (Fig. 4). A repeated measures (one within, one between) ANOVA for the trial 2 latencies, however, showed a significant group difference $\left(F_{(2,24)}\right.$ $=8.684 ; p<0.05)$ (Fig. 4), with an SNK post hoc test showing the group differences on trial 2 of the matching-to-place task to be: control $<\mathrm{RS}=\mathrm{RS}+\mathrm{CG}$. These results demonstrate superior one trial learning for a new platform location by the Long-Evans control group in the matching-to-place task.

\section{Pretraining in the swimming pool and RS lesions Place task}

A repeated measures (two within, one between) ANOVA for latency over the two testing phases showed a significant group effect $\left(F_{(1,18)}=5.215 ; p<0.05\right)$ with Long-Evans control $<$ Long-Evans RS, but no group $\times$ testing phase or group $\times$ trial interactions; it did show however, a significant group $\times$ trial $\times$ testing phase interaction $\left(F_{(9,162)}=1.932 ; p<0.05\right)$. The lack of a significant group $\times$ testing phase interaction is attributable to the fact that both groups show improvement in latencies after surgery. This is most likely the result of the carryover effects for the nonspatial components of the swimming pool task and should in no manner suggest a beneficial effect of surgery on the place task. It does suggest however, that the RS lesion does not disrupt learned nonspatial components of the swimming pool task. Evidence for this can be seen in latency scores on the first two trials after surgery (Fig. 5, Retention) where the trial 1-trial 2 performance of the control group and the RS group is very similar to the trial 1-trial 2 patterns these groups display during the matching-to-place task. In other words, even after the $10 \mathrm{~d}$ interval between testing, the LE control animals still display spatial memory for the previous platform location on the first trial of postsurgery testing and are quickly able to learn the new location, whereas the Long-Evans RS animals show no indication of memory for the old location on the first trial nor any improvement to the new location on subsequent trials. Thus, there appears to be an important difference between the groups that is masked by the carryover or savings issues that accompany a within-subject experimental design. The significant group effect and the significant group $\times$ trial $\times$ testing phase interaction also support this idea and suggest further individual ANOVAs of presurgery performance and postsurgery performance to be appropriate.

When considered alone, the presurgery performance on the place task was identical between the Long-Evans control group and the Long-Evans RS group (Fig. 5). A repeated measures (one within, one between) ANOVA for the swim latencies showed a significant trial effect $\left(F_{(9,162)}=23.548 ; p<0.05\right)$, but no group effect nor group $\times$ trial interaction, as would be expected. After surgery, however, the performance of the LongEvans control group was superior to that of the Long-Evans-RS group (Fig. 5). A repeated measures (one within, one between) ANOVA for latency on the post-surgery place task showed a significant group effect $\left(F_{(1,18)}=9.735 ; p<0.05\right)$, a significant trial effect $\left(F_{(9,162)}=4.909 ; p<0.05\right)$, and a significant group $\times$ trial interaction $\left(F_{(9,162)}=3.300 ; p<0.05\right)$.

The measure of swimming error supported the finding that the Long-Evans RS group was impaired after surgery. A repeated measures (two within, one between) ANOVA for swimming error showed a significant group difference $\left(F_{(1,18)}=16.947 ; p<0.05\right)$ and a significant group $\times$ testing phase interaction $\left(F_{(1,18)}=\right.$ 5.650; $p<0.05)$. Further analysis showed no group differences during presurgery testing, but did show a significant group effect for postsurgery testing $\left(F_{(1,18)}=17.056 ; p<0.05\right)$. 

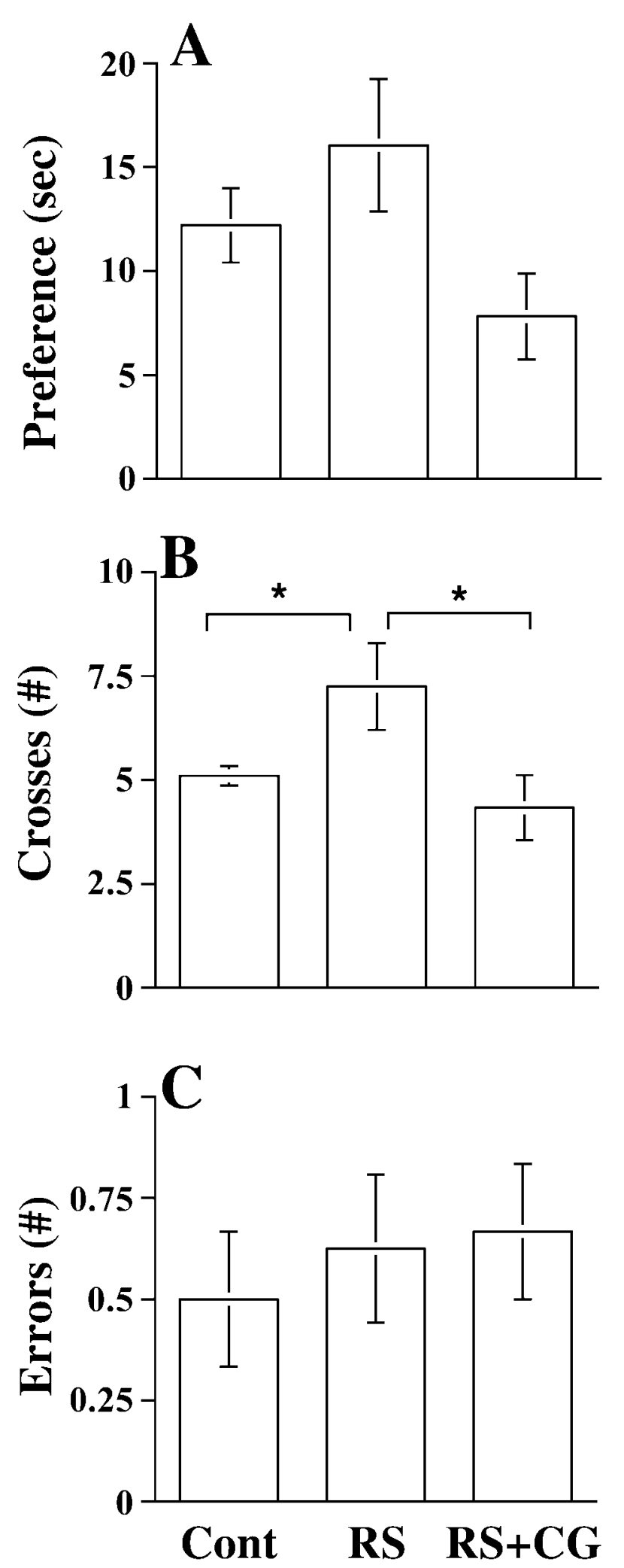

Figure 3. Mean and SE of the spatial preference score-time spent in the target quadrant relative to the other three quadrants $(0 \mathrm{sec}=$ no preference) $(A)$, the number of passes over the exact location of the hidden platform $(B)$, and the number of errors made in swimming directly to the target $(C)$, on a $60 \mathrm{sec}$ probe trial administered at the end of the place task to control, retrosplenial $(R S)$, and retrosplenial + cingulum bundle $(R S+C G)$ rats. $* p<0.05$.

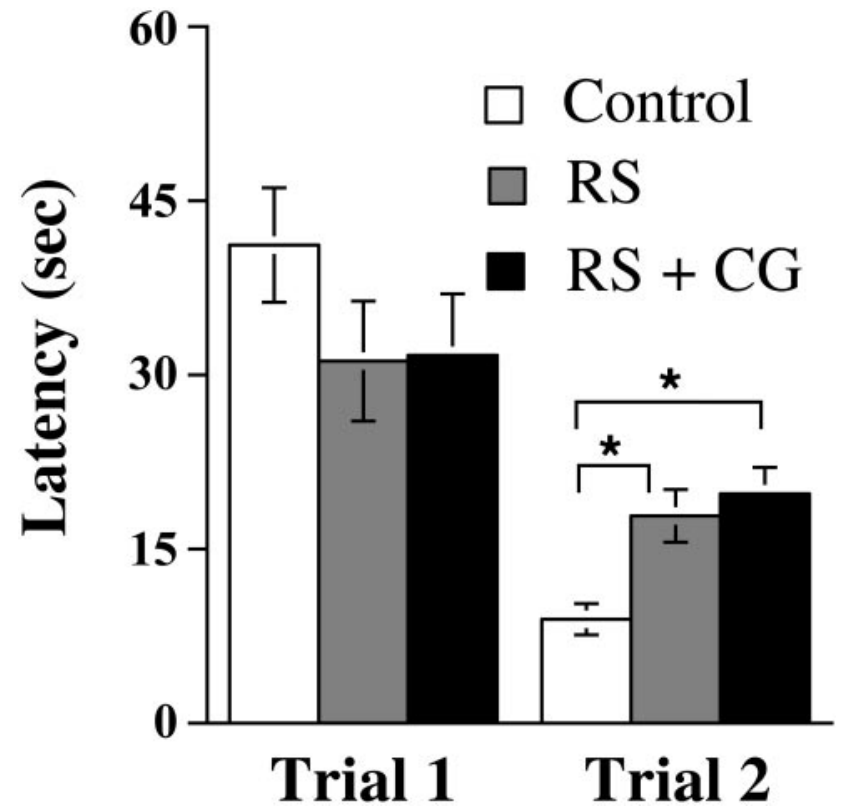

Figure 4. Latency (mean and SE) by control, retrosplenial $(R S)$, and retrosplenial + cingulum bundle $(R S+C G)$ rats over two trials averaged across platform locations on a matching-to-place task in the swimming pool. $* p<0.05$.

\section{Probe test}

A repeated measures (one within, one between) ANOVA failed to show a group $\times$ testing phase interaction for the spatial preference score (Fig. $6 A$ ) and for the number of platform crossings (Fig. $6 B$ ), but did show a significant group $\times$ testing phase interaction for swimming errors. A one-factor ANOVA showed the significant group $\times$ trial interaction to be the result of the Long-Evans RS group, making significantly more direct swimming errors after surgery $\left(F_{(1,16)}=28.000 ; p<0.05\right)$ (Fig. $\left.6 C\right)$.

\section{Matching-to-place}

Before surgery both Long-Evans groups were able to demonstrate one-trial learning on the matching-to-place task equally well (Fig. 7). After surgery, however, the RS group performed much worse on the task. These observations were confirmed by a repeated measures (three within, one between) ANOVA for latency that showed a significant group $\times$ trial $\times$ testing phase interaction $\left(F_{(1,18)}=4.837 ; p<0.05\right)$. Further analysis revealed this interaction to be the result of a significant group $\times$ trial interaction $\left(F_{(1,18)}=6.627 ; p<0.05\right)$ during the postsurgery phase of testing as the result of no one trial learning being demonstrated by the Long-Evans RS group.

\section{Long-Evans and Dark Agouti strain comparison Place task}

All three groups (Long-Evans control, Dark Agouti control, Dark Agouti RS lesion) showed some improvement over trials during the place task, however, the Long-Evans control group demonstrated a significantly more rapid decrease in latency to find the hidden platform compared with both the Dark Agouti RS group and the Dark Agouti control group (Fig. 8). Performance between the Dark Agouti control and Dark Agouti RS group was not significantly different. A repeated measures (one within, one between) ANOVA for the measure of latency showed a significant group difference $\left(F_{(2,18)}=6.549 ; p<0.05\right)$, a significant trial 


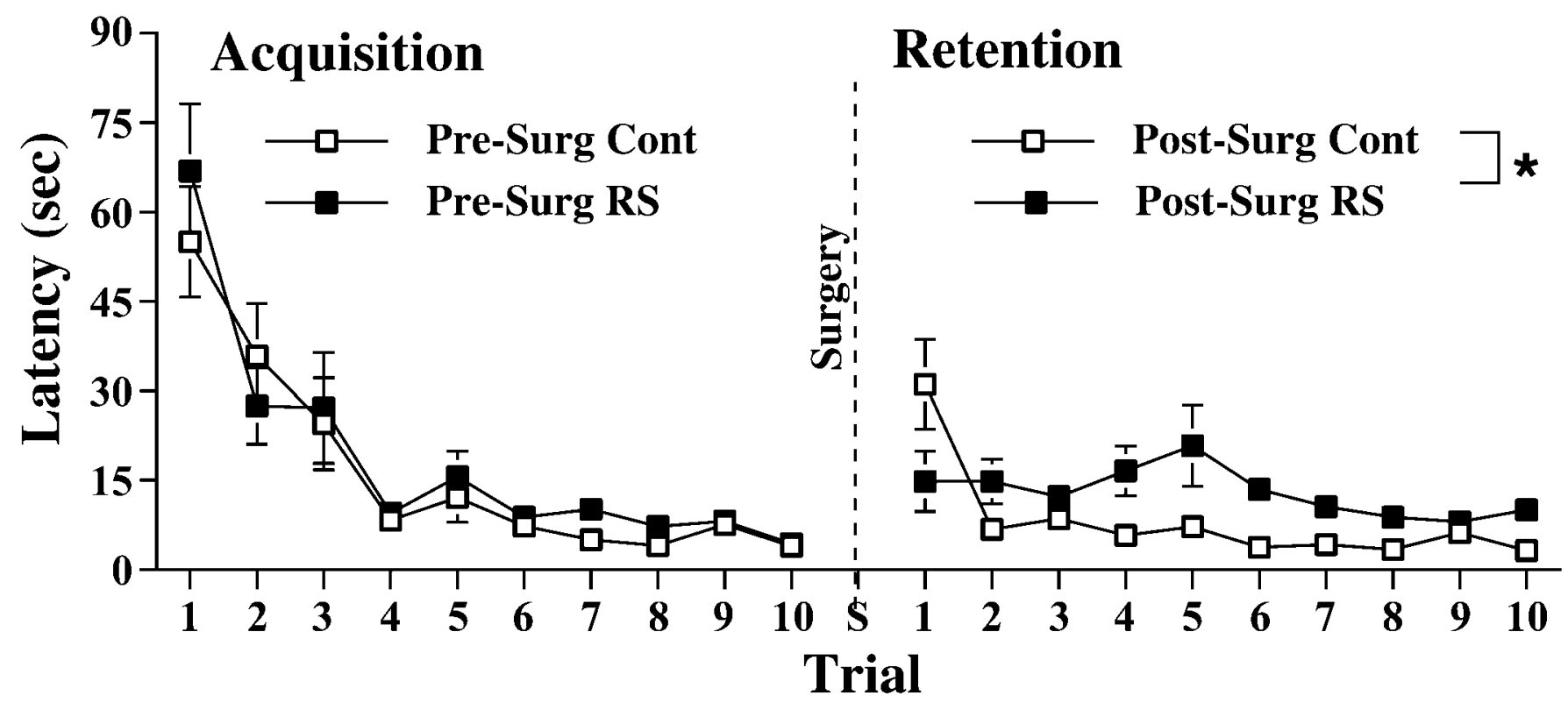

Figure 5. Latency (mean and SE) per trial by control and retrosplenial $(R S)$ rats, over 10 trials on a place task in a swimming pool both before surgery (Acquisition) and after surgery (Retention). The high control latency trial 1 score on Retention reflects their retention of the last matching-to-place trial. $* p<0.05$.

effect $\left(F_{(9,162)}=13.399 ; p<0.05\right)$, but no significant group $\times$ trial interaction. An SNK post hoc test showed the group differences as: Long-Evans $<$ Dark Agouti control = Dark Agouti RS.

Long-Evans rats also committed significantly fewer swimming errors during the swim trajectory to the platform $\left(F_{(2,18)}=9.247\right.$; $p<0.05)$ with an SNK post hoc test showing the significant differences to be: Long-Evans $<$ Dark Agouti control $=$ Dark Agouti RS.

\section{Probe test}

An ANOVA for the target quadrant preference measure on the probe trial (Fig. 9A) showed a significant group difference $\left(F_{(2,18)}=5.772 ; p<0.05\right)$. An SNK post hoc test revealed the group differences as: Dark Agouti control $>$ Dark Agouti RS. Significant group differences were also observed on the measure of target crossings $\left(F_{(2,18)}=5.860 ; p<0.05\right)$ (Fig. $\left.10 B\right)$; an SNK post hoc test revealed the differences as: Long-Evans $>$ Dark Agouti control $=$ Dark Agouti RS. There was a significant group effect for direct swim errors as well $\left(F_{(2,18)}=4.352 ; p<0.05\right)$ (Fig. 9C); an SNK post hoc test showed the differences to be: LE $<$ Dark Agouti RS. The performance of the Dark Agouti control subjects on the probe test is of interest as this group demonstrates a strong preference for the correct quadrant, yet scores significantly worse than the Long-Evans group on the number of target crossings. These results suggest that although the Dark Agouti rats have learned something about the general location of the platform in the pool, they have not learned the exact location.

\section{Matching-to-place}

Only the Long-Evans group and the Dark Agouti control group demonstrated the ability to learn the new platform location in one trial (Fig. 10). Performance by the Dark Agouti RS group was impaired relative to the other groups. A repeated measures (two within, one between) ANOVA for the measure of latency showed no significant group differences overall, but did show a significant trial effect $\left(F_{(1,18)}=40.975 ; p<0.05\right)$, and a significant group $\times$ trial interaction $\left(F_{(2,18)}=8.261 ; p<0.05\right)$.

Nonspatial errors in the place task

The Long-Evans group displayed very few nonspatial errors during the place task. Errors committed by the Long-Evans group were restricted to deflections and floating. Both the Dark Agouti control and the Dark Agouti RS groups displayed nonspatial errors of every category. An ANOVA for the nonspatial error scores showed a significant group effect (Long-Evans: mean $=0.833$; SE, 0.543; Dark Agouti control: mean, 3.889; SE, 0.696; Dark Agouti RS: mean $=4.667$; SE, 0.843; $F_{(2,18)}=6.273$; $p<0.05)$. An SNK post hoc test showed the group differences to be: Long-Evans $<$ Dark Agouti control = Dark Agouti RS.

\section{Visual acuity}

The gratings test showed that the Long-Evans control and RS groups had equal and normal visual acuity $\left(F_{(1,7)}=1.185 \mathrm{E}-\right.$ $4 ; p=0.9916)$ and that the visual acuity of Long-Evans and Dark Agouti rats was also not different $\left(F_{(1,5)}=0.457 ; p=\right.$ 0.529) (Fig. 11).

\section{DISCUSSION}

A re-examination of the role of the RS in spatial navigation in two swimming pool tasks confirmed that selective lesions limited to this structure impairs spatial behavior. Both naive animals and animals that had been pretrained before receiving lesions were impaired in the acquisition-performance of a place task and were also impaired on a matching-to-place task. Concomitant CG damage did not increase the severity of the spatial deficits. Results of other studies (Warburton et al., 1998), suggesting that RS is not involved in spatial behavior are shown to be attributable to the use of a rat strain in which the spatial impairment is masked by an innate nonspatial impairment and a failure to use a stringent testing method. Thus, the present results are definitive in indicating that RS cortex participates in spatial learning, perhaps 

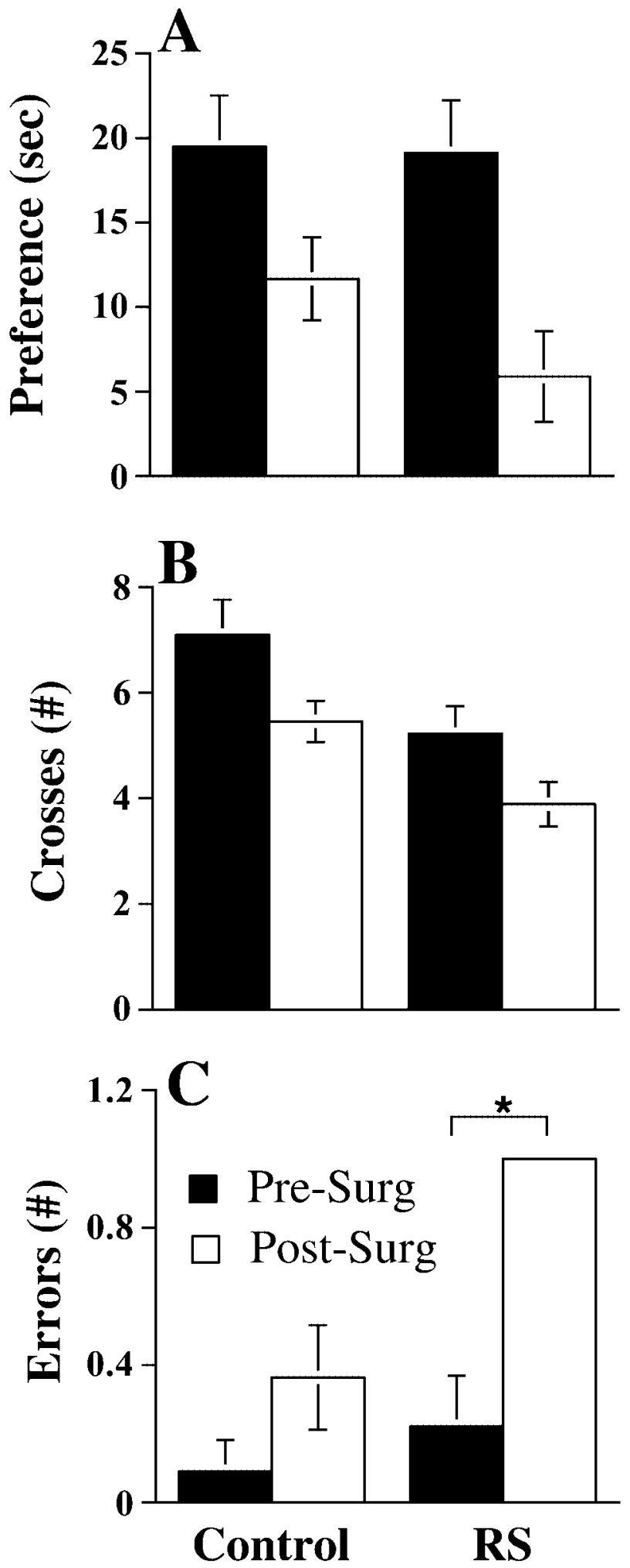

Figure 6. Presurgery and postsurgery mean and SE of the spatial preference score - time spent in the target quadrant relative to the other three quadrants $(0 \mathrm{sec}=$ no preference $)(A)$, the number of passes over the exact location of the hidden platform $(B)$, and the number of errors made in swimming directly to the target $(C)$, on a $60 \mathrm{sec}$ probe trial administered at the end of the place task to control and retrosplenial $(R S)$ rats. $* p$ $<0.05$.

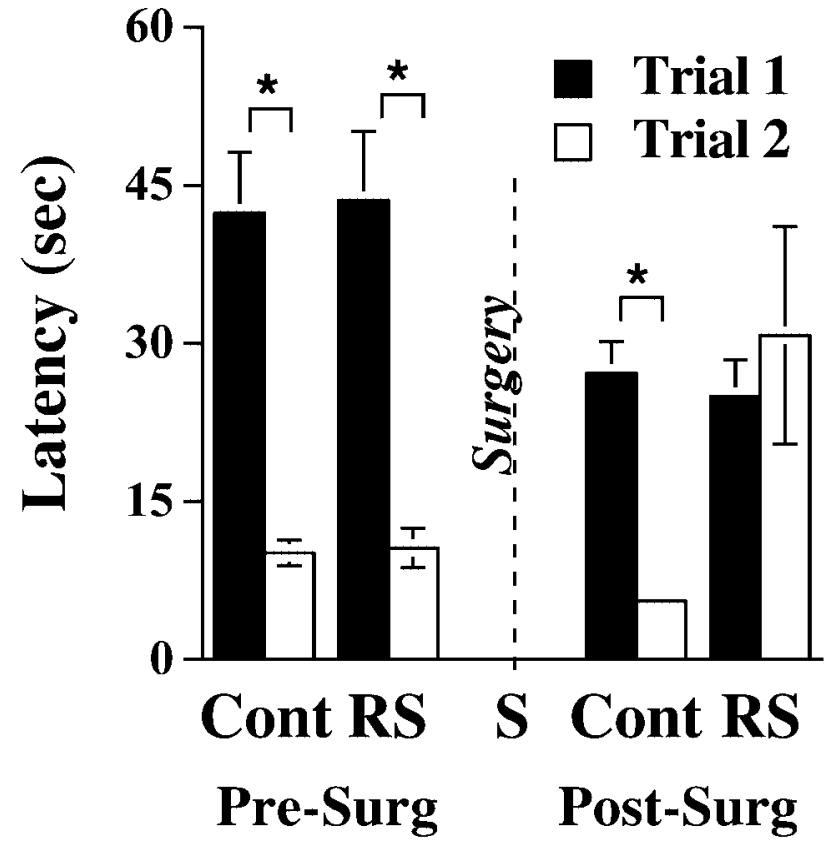

Figure 7. Presurgery and postsurgery latency (mean and SE) by control and retrosplenial $(R S)$ rats over two trials averaged across platform locations on a matching-to-place task in the swimming pool. $* p<0.05$.

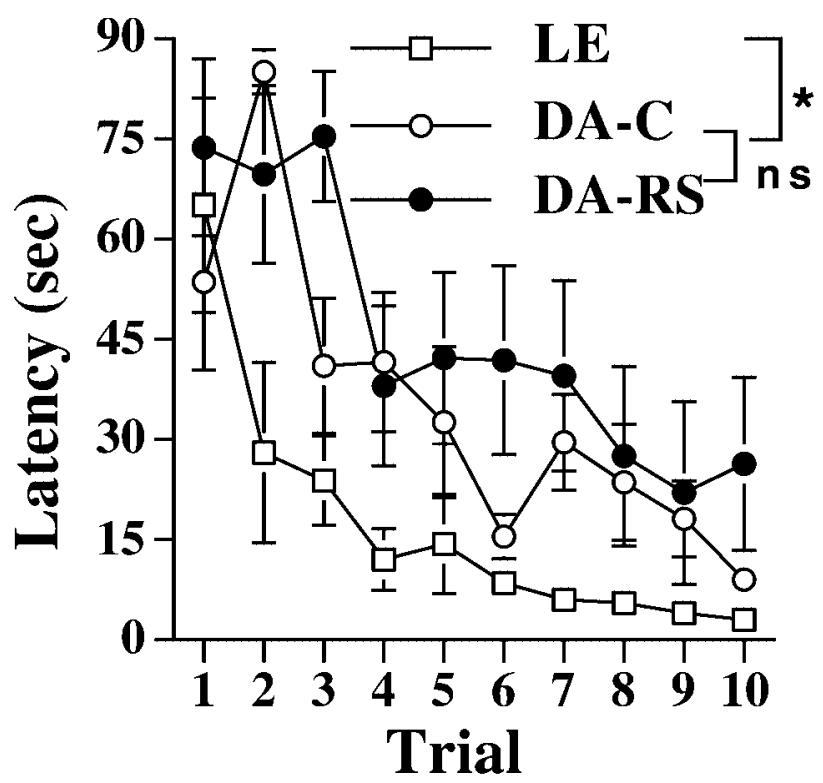

Figure 8. Latency (mean and SE) per trial by Long-Evans control $(L E)$, Dark Agouti control $(D A-C)$, and Dark Agouti retrosplenial $(D A-R S)$ rats over 10 trials on a place task in a swimming pool. $* p<0.05$.

via reciprocal anatomical connections between cortical areas and the hippocampal formation (Vogt and Miller, 1983; Pakhomova and Akopian, 1985; Wyss and Van Groen, 1992).

The present study was prompted by two seemingly irreconcilable sets of results concerning the role of RS in spatial learning. The results of one set of studies (Sutherland et al., 1988; Whishaw et al., 2001) suggest that RS is involved in spatial navigation. Rats with suction ablations of RS were impaired in learning the Morris place task (Morris et al., 1982), a task requiring that they find a stationary hidden platform in a swimming pool. The rats were 

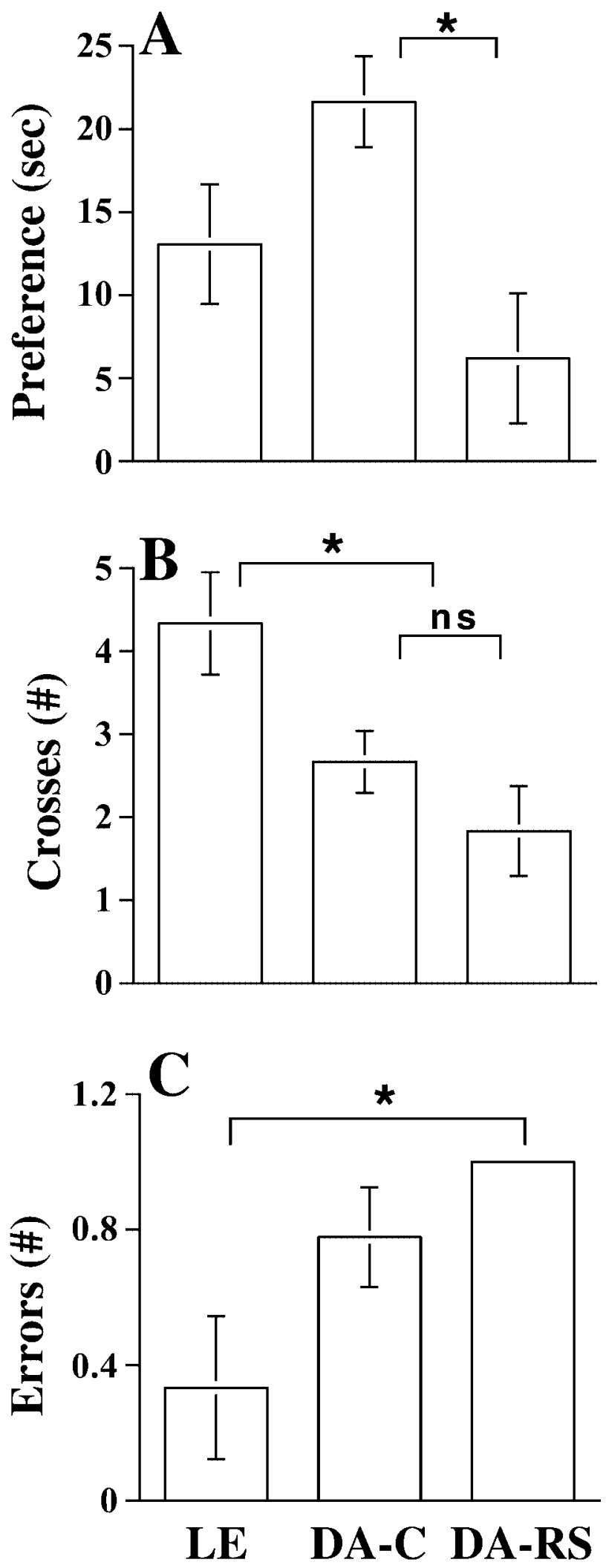

Figure 9. Mean and SE of the spatial preference score-time spent in the target quadrant relative to the other three quadrants $(0 \mathrm{sec}=$ no preference) $(A)$, the number of passes over the exact location of the hidden platform $(B)$, and the number of errors made in swimming directly to the target $(C)$, on a $60 \mathrm{sec}$ probe trial administered at the end of the place task to Long-Evans Hooded control $(L E)$, Dark Agouti control $(D A-C)$, and Dark Agouti retrosplenial $(D A-R S)$ rats. $* p<0.05$.

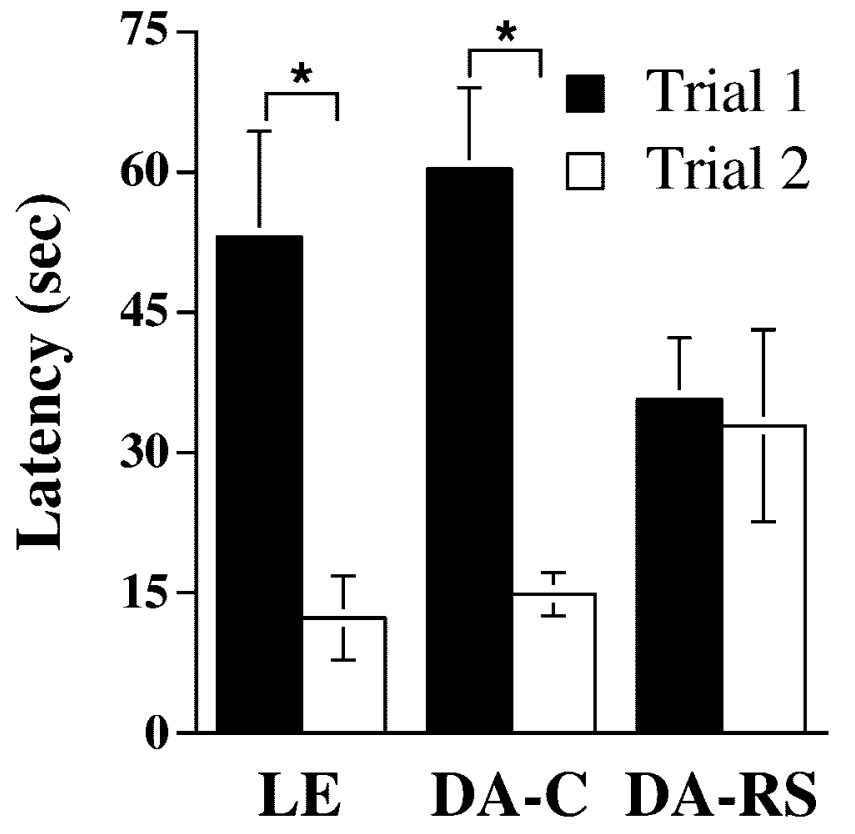

Figure 10. Latency (mean and SE) by Long-Evans control $(L E)$, Dark Agouti control $(D A-C)$, and Dark Agouti retrosplenial $(D A-R S)$ rats over two trials averaged across platform locations on a matching-to-place task in the swimming pool.

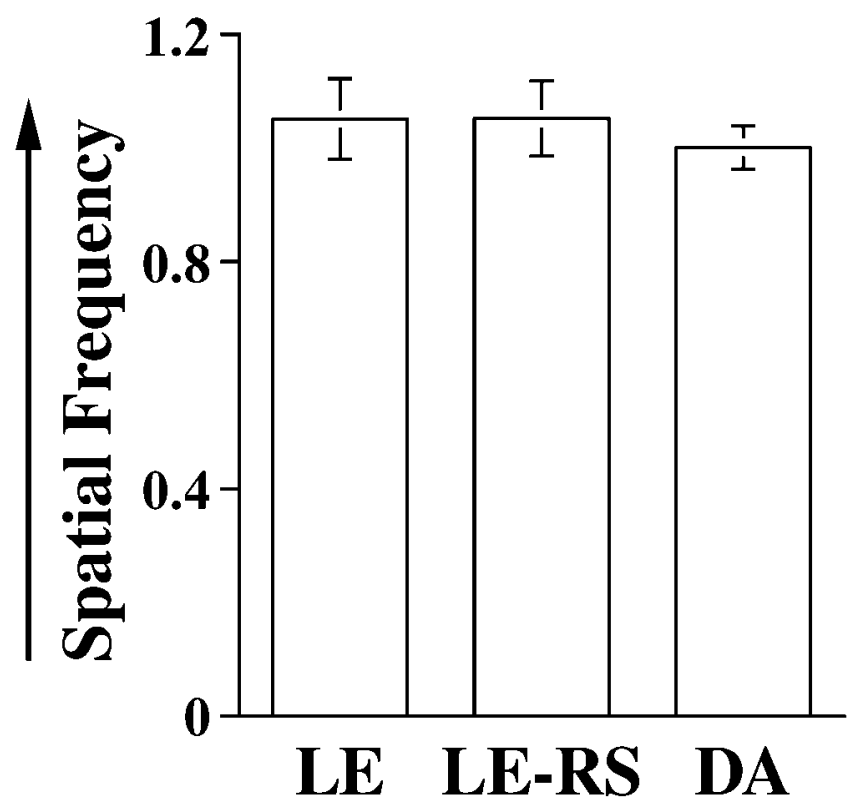

Figure 11. Mean and SE of the maximum visual acuity (measured in terms of spatial frequency or cycles per degree) demonstrated by LongEvans control $(L E)$, Long-Evans retrosplenial $(R S)$, and Dark Agouti control $(D A)$ rats. $* p<0.05$.

also impaired in the more demanding matching-to-place task that required that they learn to find the platform at a number of new locations, responses that are learned by normal rats in a single trial (Whishaw, 1985b). In the second set of studies, (Warburton et al., 1998), rats received neurotoxic lesions of the RS and were tested in only the place task. The rats are reported to have no impairment in place learning. To explain these strikingly different results, the latter studies also used animals with CG lesions alone and did find a deficit on the place task with the CG lesion. They 
suggest, therefore, that the suction ablations used by the former group produced spatial deficits because the lesions included the CG.

In the first portion of the present study we reexamined the claim (Neave et al., 1994; Aggleton et al., 1995; Warburton et al., 1998; Aggleton et al., 2000) that selective RS lesions produce no spatial deficit on the place task. We removed RS alone by stripping the meninges and restricting the suction removal to the superficial gray matter. CG was additionally removed by first removing the gray matter and then removing the most superficial portion of the white matter. Histological analysis confirmed that the desired lesions were achieved. The results of the behavioral tests showed that both RS and RS + CG lesions produced an impairment in the place task. Although impaired, both groups did show improvement with training, as indicated both by reduced times in locating the platform and heightened searches of the previously correct quadrant of the pool in a probe trial with the platform removed from the pool. The finding that RS lesions did produce a deficit in spatial learning is consistent with the first set of studies (Sutherland et al., 1988; Whishaw et al., 2001), not the second set of studies (Warburton et al., 1998). In addition, the rats with RS lesions were impaired in the matching-to-place task, a result also consistent with the first set of studies. Furthermore, these results strengthen the likelihood of RS being part of a neural circuit mediating spatial learning and memory as the working memory and reference memory impairments observed in the RS group coincide with similar memory impairments observed in other components of this circuit such as the hippocampal formation (Shapiro, 2001).

To reconcile the disparate results in these two sets of studies, we examined the possibility that the use of different strains of rats might have been a contributing factor. The first set of studies, and the first experiment of the present study, used Long-Evans rats. The rats used in the second set of studies were of the Dark Agouti strain. Our attention to possible rat strain differences was prompted by the more rapid learning displayed by the LongEvans as compared with the Dark Agouti rats in the respective studies. When we compared Dark Agouti control rats to Dark Agouti rats with RS lesions on the place task in the present study, we found no difference between the groups, and both were significantly inferior to a Long-Evans control group. That is, we confirmed that Dark Agouti rats with a RS lesion are not impaired, but we also found that the Dark Agouti strain was impaired in learning the place task relative to Long-Evans strain.

Despite the fact that we found that the Dark Agouti rats with RS lesions were not impaired in acquiring the place task relative to Dark Agouti control rats, we did find that the Dark Agouti RS group was impaired relative to the Dark Agouti control group on the matching-to-place task. Thus, we were able to demonstrate that although rats of this strain with RS lesions were not impaired in simple place acquisition relative to their control group, they were impaired in the more demanding matching-to-place task.

To understand why a rat strain difference could manifest itself in the place task and not in the matching-to-place task, it is important to recognize that neither task is selective for place learning. To learn the place task, rats must engage in considerable nonspatial learning (Whishaw, 1985a; Cain and Saucier, 1996). That is, they must learn to swim about the pool in search of an escape route, they must learn to swim away from the wall of the pool, and they must learn that when they encounter the platform it is the only escape route, etc. Only once they have acquired these procedures are they able to demonstrate place learning.
Rats may be impaired in nonspatial learning while still being able to display spatial learning (Cain and Saucier, 1996; Saucier et al., 1996; Cain, 1997; Hoh and Cain, 1997). Thus, an animal that is impaired in nonspatial learning once having acquired the nonspatial procedures during the place task may be less impaired in matching-to-place learning, which requires both the use of those procedures and spatial learning. Thus, we propose that the Dark Agouti rats are impaired in nonspatial learning, and this deficit masks the effects of an RS lesion. Nevertheless, once having acquired the nonspatial learning components of the task they are sufficiently skilled in place learning to perform the matching-toplace task, which does reveal a deficit produced by the RS lesion. Our evidence to support this hypothesis comes from the observation that several behaviors that are considered to be non-spatial learning errors, such as jumping, diving, deflections, floating, etc., (Cain and Saucier, 1996), are exhibited by the Dark Agouti rats. These behaviors were most prominent during the first few trials of the place task after which they subsided, and very rarely reappeared during the matching-to-place task.

The fact that Dark Agouti rats display a deficit in nonspatial learning raises the possibility that the RS deficits observed on the place task in our study with Long-Evans rats, and previous studies using this strain (Sutherland et al., 1988; Whishaw et al., 2001) may only be the result of a non-spatial impairment. The current study provides four lines of evidence against this possibility. First, the Long-Evans RS group that received presurgical training, a procedure that provides nonspatial information (Whishaw, 1985a; Hoh and Cain, 1997) in the swimming pool was still impaired on the place task. Second, these rats also failed to exhibit behaviors typical of nonspatial impairment (Cain and Saucier, 1996) such as thigmotaxis, failure to detect the escape platform on contact, jumping off the platform, etc. Third, all groups of rats given an RS lesion, regardless of rat strain, were impaired on matching-to-place performance. Fourth, we examined the visual acuity of control and lesion rats in a visual grating task (Prusky et al., 2000), and found visual acuity was normal. Thus, our lesion of RS did not invade primary visual cortex and so produce a visual impairment.

In the studies by the Aggleton group (Warburton et al., 1998), it is reported that selective CG lesions produced an impairment in place learning. In the present study we tested a group of rats with CG plus RS lesions and found that this combined lesion group displayed an impairment similar in size to that of the RS group. It is possible that both CG and RS lesions produce a place leaning deficit, but that possibility was not further examined in the present study.

\section{Conclusion}

In conclusion, the results of the present study demonstrate that RS lesions produce a deficit in both the place task and the matching-to-place task, thus confirming that the RS is part of a neural circuit involved in spatial behavior. The deficit was not secondary to impairments in nonspatial learning, as pretraining on both tasks before surgery did not ameliorate the deficit. This study is also the first to demonstrate that an innate impairment carried by a rat strain can mask behavioral deficits produced by a brain lesion. Nevertheless, we demonstrate that by using appropriate testing procedures, it is still possible to unmask the negative performance displayed by the rat strain thus revealing the effects of the lesion. 


\section{REFERENCES}

Aggleton JP, Neave N, Nagle S, Sahgal A (1995) A comparison of the effects of medial prefrontal, cingulate cortex, and cingulum bundle lesions on tests of spatial memory: evidence of a double dissociation between frontal and cingulum bundle contributions. $\mathbf{J}$ Neurosci 15:7270-7281.

Aggleton JP, Vann SD, Oswald CJ, Good M (2000) Identifying cortical inputs to the rat hippocampus that subserve allocentric spatial processes: a simple problem with a complex answer. Hippocampus 10:466-474.

Brown RW, Bardo MT, Mace DD, Phillips SB, Kraemer PJ (2000) D-amphetamine facilitation of Morris water task performance is blocked by eticlopride, correlated with increased dopamine synthesis in the prefrontal cortex. Behav Brain Res 114:135-143.

Cain DP (1997) Prior non-spatial pretraining eliminates sensorimotor disturbances and impairments in water maze learning caused by diazepam. Psychopharmacology (Berl) 130:313-319.

Cain DP, Saucier D (1996) The neuroscience of spatial navigation: focus on behavior yields advances. Rev Neurosci 7:215-231.

Chen LL, Lin LH, Green EJ, Barnes CA, McNaughton BL (1994a) Head-direction cells in the rat posterior cortex. I. Anatomical distribution and behavioral modulation. Exp Brain Res 101:8-23.

Chen LL, Lin LH, Barnes CA, McNaughton BL (1994b) Head-direction cells in the rat posterior cortex. II. Contributions of visual and ideothetic information to the directional firing. Exp Brain Res 101:24-34

Cho J, Sharp PE (2001) Head direction, place, movement correlates for cells in the rat retrosplenial cortex. Behav Neurosci 115:3-25.

Cooper BG, Mizumori SJ (2001) Temporary inactivation of the retrosplenial cortex causes a transient reorganization of spatial coding in the hippocampus. J Neurosci 21:3986-4001.

DeRenzi E (1982) Disorders of space exploration and cognition. New York: Wiley.

Ennaceur A, Neave N, Aggleton JP (1997) Spontaneous object recognition and object location memory in rats: the effects of lesions in the cingulate cortices, the medial prefrontal cortex, the cingulum bundle and the fornix. Exp Brain Res 113:509-519.

Geschwind N (1965) Disconnexion syndromes in animals and man. I Brain 88:237-294.

Hoh TE, Cain DP (1997) Fractionating the nonspatial pretraining effect in the water maze task. Behav Neurosci 111:1285-1291.

Kolb B, Buhrmann K, McDonald R, Sutherland RJ (1994) Dissociation of the medial prefrontal, posterior parietal, and posterior temporal cortex for spatial navigation and recognition memory in the rat. Cereb Cortex 4:664-680.

Maaswinkel H, Jarrard LE, Whishaw IQ (1999) Hippocampectomized rats are impaired in homing by path integration. Hippocampus 9:553-561.

Maguire EA (2001) The retrosplenial contribution to human navigation: a review of lesion, neuroimaging findings. Scand J Psychol 42:225-238.

Mesulam MM, Nobre AC, Kim YH, Parrish TB, Gitelman DR (2001) Heterogeneity of cingulate contributions to spatial attention. NeuroImage 13:1065-1072.

Morris RG, Garrud P, Rawlins JN, O'Keefe J (1982) Place navigation impaired in rats with hippocampal lesions. Nature 297:681-683.

Neave N, Lloyd S, Sahgal A, Aggleton JP (1994) Lack of effect of lesions in the anterior cingulate cortex and retrosplenial cortex on certain tests of spatial memory in the rat. Behav Brain Res 65:89-101.

Neave N, Nagle S, Aggleton JP (1997) Evidence for the involvement of the mammillary bodies and cingulum bundle in allocentric spatial processing by rats. Eur J Neurosci 9:941-955.

Pakhomova AS, Akopian EV (1985) Direct connections between the hippocampus and the retrosplenial cortex in the rat. Neirofiziologiia 17:102-107.

Palmer MJ, Mumby DG, Cooper BG, Robison SE, Longstreet RR, Sutherland RJ (1993) Medial prefrontal cortex and retrosplenial cortex make different contributions to memory for objects and places. Soc Neurosci Abstr, 19:364.

Pandya DN, Yeterian EH (1984) Proposed neural circuitry for spatial memory in the primate brain. Neuropsychologia 22:109-122.

Papez JW (1937) A proposed mechanism of emotion. Arch Neurol Psychiatry 38:725-734.

Pohl W (1973) Dissociation of spatial discrimination deficits following frontal and parietal lesions in monkeys. J Comp Physiol Psychol 82:227-239.

Prusky GT, West PW, Douglas RM (2000) Behavioral assessment of visual acuity in mice and rats. Vision Res 40:2201-2209.

Saucier D, Hargreaves EL, Boon F, Vanderwolf CH, Cain DP (1996) Detailed behavioral analysis of water maze acquisition under systemic NMDA or muscarinic antagonism: nonspatial pretraining eliminates spatial learning deficits. Behav Neurosci 110:103-116.

Shapiro M 2001 Plasticity, hippocampal place cells, cognitive maps. Arch Neurol 58:874-881.

Sutherland RJ, Hoesing JM (1993) Posterior cingulate cortex and spatial memory: a microlimnological analysis. In: Neurobiology of cingulate cortex (Vogt BA, Gabriel M, eds), pp 461-477. Boston: Birkhauser.

Sutherland RJ, Whishaw IQ, Kolb B (1983) A behavioural analysis of spatial localization following electrolytic, kainate- or colchicineinduced damage to the hippocampal formation in the rat. Behav Brain Res 7:133-153.

Sutherland RJ, Whishaw IQ, Kolb B (1988) Contributions of cingulate cortex to two forms of spatial learning and memory. J Neurosci 8:1863-1872.

Vogt BA, Miller MW (1983) Cortical connections between rat cingulate cortex and visual, motor, and postsubicular cortices. J Comp Neurol 216:192-210.

Warburton EC, Aggleton JP, Muir JL (1998) Comparing the effects of selective cingulate cortex lesions and cingulum bundle lesions on water maze performance by rats. Eur J Neurosci 10:622-634.

Whishaw IQ (1985a) Cholinergic receptor blockade in the rat impairs locale but not taxon strategies for place navigation in a swimming pool. Behav Neurosci 99:979-1005

Whishaw IQ (1985b) Formation of a place learning-set by the rat: a new paradigm for neurobehavioral studies. Physiol Behav 35:139-143.

Whishaw IQ, Jarrard LE (1995) Similarities vs. differences in place learning and circadian activity in rats after fimbria-fornix section or ibotenate removal of hippocampal cells. Hippocampus 5:595-604.

Whishaw IQ, Maaswinkel H, Gonzalez CL, Kolb B (2001) Deficits in allothetic, idiothetic spatial behavior in rats with posterior cingulate cortex lesions. Behav Brain Res 118:67-76.

Winer BJ (1962) Statistical principles in experimental design. New York: McGraw-Hill.

Wozniak DF, Brosnan-Watters G, Nardi A, McEwen M, Corso TD, Olney JW, Fix AS (1996) MK-801 neurotoxicity in male mice: histologic effects and chronic impairment in spatial learning. Brain Res 707:165-179.

Wyss JM, Van Groen T (1992) Connections between the retrosplenial cortex and the hippocampal formation in the rat: a review. Hippocampus $2: 1-11$. 